\title{
Review
}

\section{Dopamine Cell Therapy: From Cell Replacement to Circuitry Repair}

\author{
Anders Björklund* and Malin Parmar \\ Department of Experimental Medical Science, Developmental and Regenerative Neurobiology, Wallenberg \\ Neuroscience Center, Lund University, Lund, Sweden
}

Accepted 6 March 2021

Pre-press 29 March 2021

\begin{abstract}
Cell therapy for Parkinson's disease (PD) is aimed to replace the degenerated midbrain dopamine (mDA) neurons and restore DA neurotransmission in the denervated forebrain targets. A limitation of the intrastriatal grafting approach, which is currently used in clinical trials, is that the mDA neurons are implanted into the target area, in most cases the putamen, and not in the ventral midbrain where they normally reside. This ectopic location of the cells may limit their functionality due to the lack of appropriate afferent regulation from the host. Homotopic transplantation, into the substantia nigra, is now being pursued in rodent PD models as a way to achieve more complete circuitry repair. Intranigral grafts of mDA neurons, derived from human embryonic stem cells, have the capacity to re-establish the nigrostriatal and mesolimbic pathways in their entirety and restore dense functional innervations in striatal, limbic and cortical areas. Tracing of host afferent inputs using the rabies tracing technique shows that the afferent connectivity of grafts implanted in the nigra matches closely that of the intrinsic mDA system, suggesting a degree of circuitry reconstruction that exceeds what has been achieved before. This approach holds great promise, but to match the larger size of the human brain, and the 10 times greater distance between substantia nigra and its forebrain targets, it may be necessary to find ways to improve the growth capacity of the grafted $\mathrm{mDA}$ neurons, pointing to a combined approach where growth promoting factors are used to enhance the performance of $\mathrm{mDA}$ neuron grafts.
\end{abstract}

Keywords: Parkinson's disease, embryonic stem cells, transplantation, connectivity

Transplantation of midbrain dopamine (mDA) neurons derived from pluripotent stem cells are currently being explored in clinical trials in patients with Parkinson's disease (PD) with the aim to restore DA neurotransmission in the DA depleted striatum $[1,2]$. The current approach has an obvious limitation in that the cells are ectopically implanted in the DA target area rather into the substantia nigra where they normally reside (see Fig. 1A). It is clear from both experimental studies and studies in patients that the ectopic, intrastriatal transplants are spontaneously

\footnotetext{
${ }^{*}$ Correspondence to: Anders Björklund, Wallenberg Neuroscience Center, BMCA11, 22184 Lund, Sweden. Tel.: +4670 3146761; E-mail: anders.bjorklund@med.lu.se.
}

active and release DA in a tonic, physiological manner. Although this is sufficient to provide significant improvement in motor function, not all aspects of the DA deficiency syndrome seen in DA lesioned animals and PD patients are well restored in this grafting paradigm $[3,4]$.

This raises the question whether mDA neuron grafts placed homotopically, in the substantia nigra (Fig. 1B), would be able to provide more extensive restoration of the damaged nigro-striatal circuitry and thus more complete functional recovery. This was for a long time considered unrealistic due to the long distance between nigra and striatum and the complexity of the afferent connectivity that would have to be restored. The initial attempts made along this line 

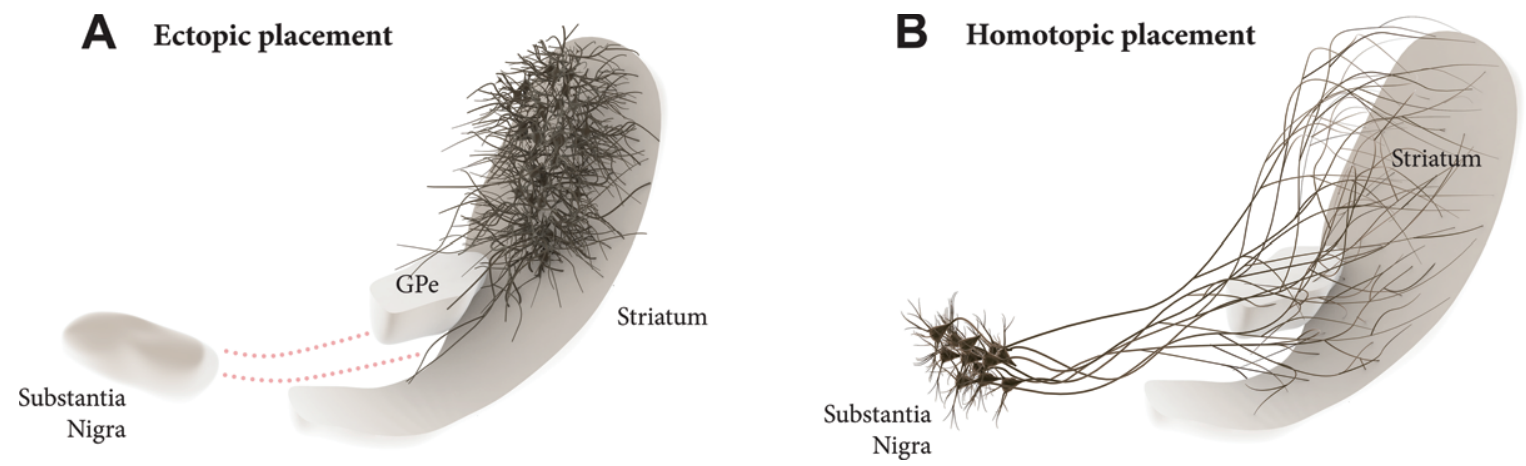

Fig. 1. Dopamine (DA) neuron transplantation seeks to replace the lost midbrain DA neurons and restore DA neurotransmission in the DA-depleted striatum. In the clinical trials conducted so far the DA neurons are transplanted into the striatum (A), i.e., the area where DA is released. This ectopic location of the cells may limit their functionality due to the lack of appropriate afferent regulation from the host. In the alternative approach discussed here the cells are implanted homotopically into the substantia nigra (B), i.e., the site where the nigrostriatal DA neurons normally reside, making it possible to achieve more complete restoration of efferent and afferent graft-host connectivity.

were also discouraging. Promising progress, however, has been made during the last decade, revealing a remarkable ability of pre-specified neuronal precursors to integrate into host circuitry and extend axons over large distances in the adult brain, illustrated, in particular, by the highly specific connectivity that has been achieved from neocortical neuron precursors implanted into lesioned neocortex in rodents [5-8]. Similar promising results have been obtained using intranigral transplants of human fetal or embryonic stem cell (hESC) derived mDA neurons.

\section{EARLY STUDIES}

In studies performed in the 1990s, the performance of intranigral grafts of fetal rat mDA neurons were explored in unilaterally 6-hydroxydopamine (6OHDA) lesioned rats. The neurons were seen to survive well but the extension of tyrosine hydroxylase (TH) positive axons toward the striatum was very limited or entirely absent. Interestingly, however, the intranigral grafts had significant functional impact even in the absence of striatal connectivity, seen as a recovery of both drug-induced and spontaneous motor behavior, such as forelimb stepping and movement initiation time $[9,10]$.

These findings highlighted the often-neglected fact that DA released locally from dendrites act to regulate GABA release via activation of DA D1 receptors located on GABAergic terminals in the pars reticulata. In rodent models at least, DA produced in the nigra contributes to the functional effect of systemic L-DOPA, adding to the effects elicited in the striatum [11]. Inspired by these findings Mendez et al. [12] used a combination of intraputaminal and intranigral grafting in four PD patients. Although these patients showed very good clinical outcome, and good survival of both the intranigral and intraputaminal grafts, the design of the study and the limited number of patients did not allow any conclusion as to the relative contribution of the intranigral grafts.

\section{RECONSTRUCTION OF THE NIGRO-STRIATAL AND MESOLIMBIC PATHWAYS}

During the last decade the introduction of new and more powerful axonal tracing techniques has led to a revival of the intranigral grafting approach. Using dissected fetal ventral mesencephalon (VM) from GFP reporter mice, Gaillard et al. [13] and Thompson et al. [14] were the first to show reformation of a complete and functional nigrostriatal pathway in 6-OHDA lesioned mice from mDA neurons grafted into the nigra. Subsequently, even more extensive forebrain connectivity from neurons grafted to the nigra in 6-OHDA lesioned rodents has been obtained using fetal human and hESC-derived mDA neurons [15-18]. The specificity of graft-to-host connectivity observed in these studies is quite remarkable: Cellular markers indicate that both major types of mDA neurons, A9 and A10, are contained in these grafts, and their projections are quite similar to those of the endogenous counterparts, resulting in dense innervations of striatal, limbic and cortical areas normally innervated by the mDA neurons (Fig. 1B). This outgrowth pattern is clearly cell-type specific: intranigral grafts of hESC-derived glutamatergic neurons with 
a forebrain phenotype innervate cortical and ventral forebrain areas but avoid completely the caudateputamen $[15,18]$.

Interestingly, the areas innervated by ectopic intrastriatal mDA neuron grafts $[14,19]$ match quite well those innervated by the intranigral grafts (Fig. 1B), showing that the targeting is independent of the location of the cells. The outgrowing axons are clearly able to seek out their appropriate targets from any direction, suggesting the presence of highly specific axon guidance and target recognition mechanisms in the DA-denervated forebrain. This is further supported by the observation that the extension of axons from intranigral fetal mDA neuron grafts is almost completely blocked if the intrinsic projection is left intact [14].

Tests of drug-induced and spontaneous motor behaviors indicate that the newly established connections are functional and restored gradually over a period of 3-4 months, which matches well the timecourse of axonal outgrowth and generation of axonal terminals in the striatal target area $[16,18]$. Recovery of DA neurotransmission is further supported by studies using chemogenetic tools (DREADDs), applied initially to intrastriatal grafts [20, 21], and more recently also to the study of hESC-derived mDA neurons grafted to the nigra [18]. In both cases DREADD-induced inhibition of the grafted mDA neurons was shown to reverse the graft induced motor recovery, an effect shown also using optogenetic silencing [22]. DREADD-induced activation induced the opposite effect, i.e., a further enhancement of the graft-induced functional improvement. In the study by Chen et al. [21], this enhancement effect was blocked by pretreatment with DA receptor antagonists. These data show that intranigral mDA neuron grafts are as functional as cells grafted to the striatum. In both cases, functional recovery is critically dependent on the recovery of DA neurotransmission in the innervated forebrain targets, although it is possible that DA released locally in the nigra may contribute as well (see above).

Similar to fetal VM grafts, the hESC-derived grafts also contain non-DA neurons. Their axons, visualized using a combination of TH and human NCAM antibodies, project like the TH-positive ones to wide and partially overlapping areas of the forebrain $[14,23$, 24]. Their projection pattern is similar to the midbrain GABA and glutamate neurons that normally reside in the VTA. Around $45 \%$ of the neurons in the VTA are known to be non-dopaminergic, most of them GABAergic or glutamatergic [24-26]. Like the DA neurons, they project widely to the forebrain, and in rats and mice, the VTA projections to several limbic and cortical areas are more than $50 \%$ nondopaminergic [27], which is in line with the TH and non-TH innervations we see in limbic and cortical regions in transplanted animals [14, 15]. It seems likely therefore that this mixed composition, which is an essential feature of VTA also in the human brain [27], is retained also in the hESC-derived transplants.

\section{TRACING OF HOST-TO-GRAFT CONNECTIVITY}

Previous electrophysiological and optogenetic studies have shown that grafted fetal mDA neurons are spontaneously active and receive both excitatory and inhibitory inputs from the host [22, 28, 29], but it was the introduction of the monosynaptic rabies tracing technique that made it possible to investigate the origins and extent of host afferents in their entirety. In this method the grafted cells are equipped, prior to grafting, with the TVA receptor (for selective infection of the pseudotyped rabies vector) and the rabies glycoprotein (which allows spread retrogradely across the synapse). This allows the fluorescent label in the pseudotyped rabies vector to be transferred selectively from the graft to all host (and graft) neurons synapsing onto the grafted cells. Using this approach, it has been possible to map and compare the afferent inputs to grafts placed in either their normal, homotopic, location in the nigra, or ectopically in the striatum, allowing also a direct comparison with the pattern of afferents to the endogenous midbrain DA neurons $[15,18,30]$.

These studies show that the afferent connectivity of intranigral grafts is strikingly similar to that of the endogenous midbrain DA neurons, with major inputs from the striatum (GABAergic projection neurons), sensorimotor cortex (glutamatergic CTIP2 ${ }^{+}$ and $\mathrm{SATB}^{+}$projection neurons), and external globus pallidus (GABAergic pallido-nigral projection neurons), as well as from the central amygdala, bed nucleus of the stria terminalis, lateral and periventricular hypothalamus, and dorsal raphe [15, 16, 18]. The afferents to intrastriatal grafts originate to a large extent from the same host brain areas, striatum, sensorimotor cortex and external globus pallidus in particular, but they are also different: The intrastriatal grafts receive abundant afferents from the parafascicular and mediodorsal thalamus, which are absent from the intranigral grafts, and fewer inputs from 
A Human dopamine system

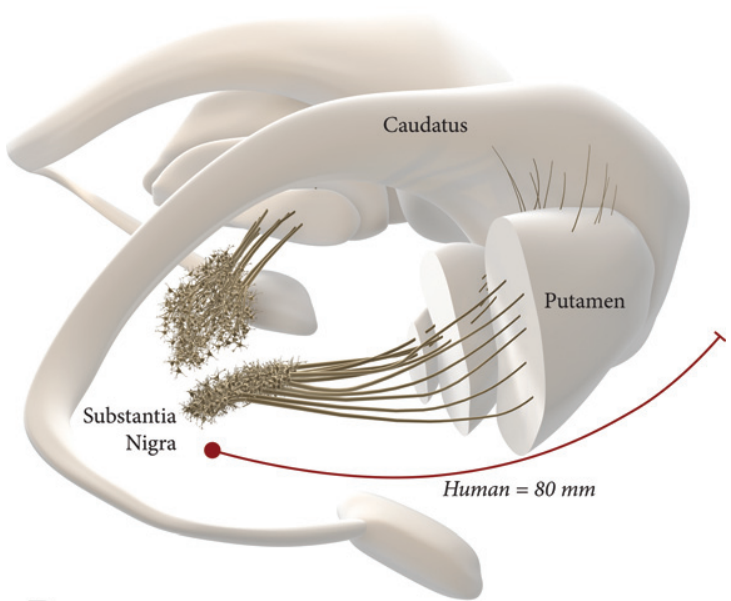

B Rat dopamine system

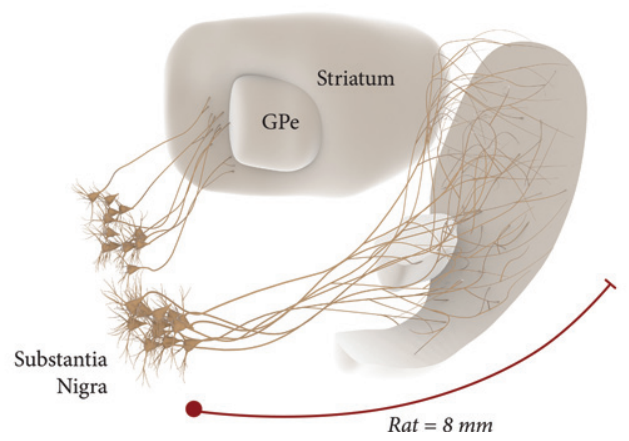

Fig. 2. Intranigral grafting is attractive as a way to achieve more complete circuitry repair, more refined functional regulation of the grafted neurons, and more widespread reinnervation of the DA-deficient forebrain areas. The use of this approach in the human brain, however, is a challenge due to the 10-fold greater length of the human DA system (A), compared to the one in the rat brain (B). This may require a combined therapeutic approach where growth promoting factors are used to increase the growth capacity of the grafted mDA neurons.

hypothalamus and caudal brainstem, which innervate the intranigral grafts, as is the case also for the host mDA neurons $[15,18,31]$.

The close-to-normal efferent and afferent connectivity of the intranigral grafts indicates a remarkable degree of circuitry reconstruction that far exceeds what has been possible to achieve with ectopic intrastriatal grafts. Nevertheless, the extent of afferent inputs to intrastriatal grafts is intriguing and challenges the common view that the functional impact of ectopically placed DA neuron grafts is mediated by autoregulated, tonic activity in the absence of normal regulatory inputs. Despite their ectopic location the intrastriatal grafts receive excitatory and inhibitory inputs from cortical, striatal and pallidal neurons that are known to regulate the function of the endogenous nigral DA neurons. It seems possible that the intrastriatal grafts could receive inputs, via branching collaterals, from functionally appropriate subtypes of excitatory (cortical) and inhibitory (striatal and pallidal) neurons in the host. In support of this idea, Adler et al. [15] could show that the synaptic inputs to intrastriatal grafts are derived, at least in part, from the same cortical, striatal and pallidal neurons that innervate the host substantia nigra.

\section{CLINICAL PERSPECTIVE}

The impressive results obtained in rodent models raise the question whether intranigral transplantation should be explored also in patients. This approach is attractive, not only as a way to achieve more complete circuitry repair and more refined functional regulation of the grafted neurons, but also as a way to obtain more widespread reinnervation of the 
Table 1

Take home message

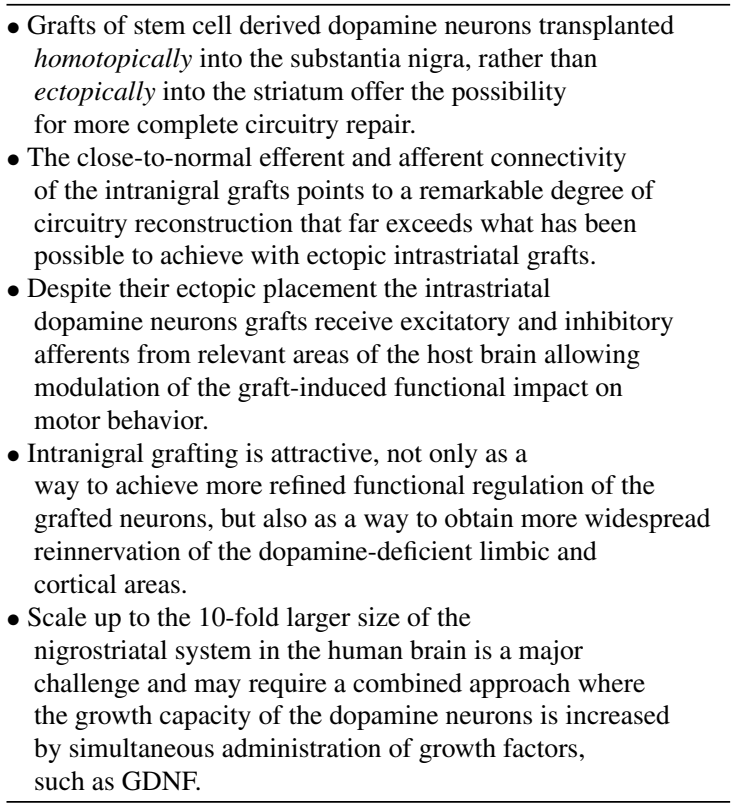

DA-deficient forebrain areas. In contrast to intrastriatal grafting where expansion of innervation into additional forebrain targets, such as caudate nucleus, nucleus accumbens and prefrontal cortex, requires an increased number of implantation sites (thus more needle tracts), the intranigral approach would make it possible to obtain widespread reinnervation from a single implantation site. Since the progressive loss of DA innervation in limbic and cortical areas is likely to contribute to the development on non-motor symptoms - as suggested by the improvements in sleep, fatigue, mood and cognition obtained with continuous intestinal L-DOPA infusions [32, 33] -it seems possible that the intranigral grafting approach, resulting in reinnervation of both striatal, limbic, and cortical territories, could have a broader impact on PD symptoms than grafts limited to the striatum.

The scale up to the much larger human brain is a challenge, however. The nigrostriatal pathway in the rat is about $4-5 \mathrm{~mm}$ long, and the maximum extension of axons that has been observed from intranigral grafts, reaching anteromedial frontal cortex in the rat, is about $8-9 \mathrm{~mm}$. In humans, the distance from the nigra to the rostral parts of the caudate/putamen and nc. accumbens is about $6-7 \mathrm{~cm}$, and a further $1-2 \mathrm{~cm}$ to the anteromedial frontal cortex, i.e., around 10 times larger than in the rat (Fig. 2). To match the larger size of the human brain, therefore, it may be necessary to find ways to improve the growth capacity of the grafted mDA neurons. Efforts along this line have been focused on the combination with growth factors (GDNF in particular) [14, 34, 35] and/or chemoattractants such as Netrin-1 [34, 36, 37]. A recent study from the Thompson/Parish lab is particularly promising in this regard, showing a prominent growth-stimulating effect of GDNF on grafted hESC-derived mDA neurons, resulting in an increased reinnervation of remote targets (such as the perirhinal and cingulate cortex), accompanied by accelerated functional recovery [38]. This points to the possibility of a combined therapeutic approach where neurotrophic/growth attractant factors are used to increase the performance of intranigrally grafted mDA neurons. Such a dual approach, if successfully developed, may have the additional advantage in helping to preserve what is left of the intrinsic DA system and thereby providing additional support to the efficacy and long-term clinical outcome of DA neuron grafts. In a future scenario, maximal longterm impact of a fully circuit-integrated intranigral $\mathrm{mDA}$ neuron graft would be achieved when used in combination with a protective intervention blocking the progression of the underlying disease.

\section{ACKNOWLEDGMENTS}

We thank Bengt Mattsson for excellent help with the preparation of the figures.

\section{CONFLICT OF INTEREST}

$\mathrm{AB}$ has no potential conflict of interest. MP is the owner of Parmar Cells AB and co-inventor of the U.S. patent application 15/093,927 owned by Biolamina $\mathrm{AB}$ and EP17181588 owned by Miltenyi Biotec.

\section{REFERENCES}

[1] Kim TW, Koo SY, Studer L (2020) Pluripotent stem cell therapies for Parkinson disease: Present challenges and future opportunities. Front Cell Dev Biol 8, 729.

[2] Parmar M, Grealish S, Henchcliffe C (2020) The future of stem cell therapies for Parkinson disease. Nat Rev Neurosci 21, 103-115.

[3] Barker RA, Barrett J, Mason SL, Bjorklund A (2013) Fetal dopaminergic transplantation trials and the future of neural grafting in Parkinson's disease. Lancet Neurol 12, 84-91.

[4] Bjorklund A, Parmar M (2020) Neuronal replacement as a tool for basal ganglia circuitry repair: 40 years in perspective. Front Cell Neurosci 14, 146. 
[5] Espuny-Camacho I, Michelsen KA, Linaro D, Bilheu A, Acosta-Verdugo S, Herpoel A, Giugliano M, Gaillard A, Vanderhaeghen P (2018) Human pluripotent stem-cellderived cortical neurons integrate functionally into the lesioned adult murine visual cortex in an area-specific way. Cell Rep 23, 2732-2743.

[6] Falkner S, Grade S, Dimou L, Conzelmann KK, Bonhoeffer T, Gotz M, Hubener M (2016) Transplanted embryonic neurons integrate into adult neocortical circuits. Nature 539, 248-253.

[7] Gronning Hansen M, Laterza C, Palma-Tortosa S, Kvist G, Monni E, Tsupykov O, Tornero D, Uoshima N, Soriano J, Bengzon J, Martino G, Skibo G, Lindvall O, Kokaia Z (2020) Grafted human pluripotent stem cell-derived cortical neurons integrate into adult human cortical neural circuitry. Stem Cells Transl Med 9, 1365-1377.

[8] Linaro D, Vermaercke B, Iwata R, Ramaswamy A, LibePhilippot B, Boubakar L, Davis BA, Wierda K, Davie K, Poovathingal S, Penttila PA, Bilheu A, De Bruyne L, Gall D, Conzelmann KK, Bonin V, Vanderhaeghen P (2019) Xenotransplanted human cortical neurons reveal species-specific development and functional integration into mouse visual circuits. Neuron 104, 972-986 e976.

[9] Mukhida K, Baker KA, Sadi D, Mendez I (2001) Enhancement of sensorimotor behavioral recovery in hemiparkinsonian rats with intrastriatal, intranigral, and intrasubthalamic nucleus dopaminergic transplants. $J \mathrm{Neu}$ rosci 21, 3521-3530.

[10] Winkler C, Bentlage C, Nikkhah G, Samii M, Bjorklund A (1999) Intranigral transplants of GABA-rich striatal tissue induce behavioral recovery in the rat Parkinson model and promote the effects obtained by intrastriatal dopaminergic transplants. Exp Neurol 155, 165-186.

[11] Robertson HA (1992) Dopamine receptor interactions: Some implications for the treatment of Parkinson's disease. Trends Neurosci 15, 201-206.

[12] Mendez I, Sanchez-Pernaute R, Cooper O, Vinuela A, Ferrari D, Bjorklund L, Dagher A, Isacson O (2005) Cell type analysis of functional fetal dopamine cell suspension transplants in the striatum and substantia nigra of patients with Parkinson's disease. Brain 128, 1498-1510.

[13] Gaillard A, Decressac M, Frappe I, Fernagut PO, Prestoz L, Besnard S, Jaber M (2009) Anatomical and functional reconstruction of the nigrostriatal pathway by intranigral transplants. Neurobiol Dis 35, 477-488.

[14] Thompson LH, Grealish S, Kirik D, Bjorklund A (2009) Reconstruction of the nigrostriatal dopamine pathway in the adult mouse brain. Eur J Neurosci 30, 625-638.

[15] Adler AF, Cardoso T, Nolbrant S, Mattsson B, Hoban DB, Jarl U, Wahlestedt JN, Grealish S, Bjorklund A, Parmar M (2019) hESC-derived dopaminergic transplants integrate into basal ganglia circuitry in a preclinical model of Parkinson's disease. Cell Rep 28, 3462-3473 e3465.

[16] Cardoso T, Adler AF, Mattsson B, Hoban DB, Nolbrant S, Wahlestedt JN, Kirkeby A, Grealish S, Bjorklund A, Parmar M (2018) Target-specific forebrain projections and appropriate synaptic inputs of hESC-derived dopamine neurons grafted to the midbrain of parkinsonian rats. J Comp Neurol 526, 2133-2146.

[17] Grealish S, Diguet E, Kirkeby A, Mattsson B, Heuer A, Bramoulle Y, Van Camp N, Perrier AL, Hantraye P, Bjorklund A, Parmar M (2014) Human ESC-derived dopamine neurons show similar preclinical efficacy and potency to fetal neurons when grafted in a rat model of Parkinson's disease. Cell Stem Cell 15, 653-665.
[18] Xiong M, Tao Y, Gao Q, Feng B, Yan W, Zhou Y, Kotsonis TA, Yuan T, You Z, Wu Z, Xi J, Haberman A, Graham J, Block J, Zhou W, Chen Y, Zhang SC (2021) Human stem cell-derived neurons repair circuits and restore neural function. Cell Stem Cell 28, 112-126 e116.

[19] de Luzy IR, Niclis JC, Gantner CW, Kauhausen JA, Hunt CPJ, Ermine C, Pouton CW, Thompson LH, Parish CL (2019) Isolation of LMX1a ventral midbrain progenitors improves the safety and predictability of human pluripotent stem cell-derived neural transplants in parkinsonian disease. J Neurosci 39, 9521-9531.

[20] Aldrin-Kirk P, Heuer A, Wang G, Mattsson B, Lundblad M, Parmar M, Bjorklund T (2016) DREADD modulation of transplanted DA neurons reveals a novel parkinsonian dyskinesia mechanism mediated by the serotonin 5-HT6 receptor. Neuron 90, 955-968.

[21] Chen Y, Xiong M, Dong Y, Haberman A, Cao J, Liu H, Zhou W, Zhang SC (2016) Chemical control of grafted human PSC-derived neurons in a mouse model of Parkinson's disease. Cell Stem Cell 18, 817-826.

[22] Steinbeck JA, Choi SJ, Mrejeru A, Ganat Y, Deisseroth K, Sulzer D, Mosharov EV, Studer L (2015) Optogenetics enables functional analysis of human embryonic stem cell-derived grafts in a Parkinson's disease model. Nat Biotechnol 33, 204-209.

[23] Niclis JC, Gantner CW, Hunt CPJ, Kauhausen JA, Durnall JC, Haynes JM, Pouton CW, Parish CL, Thompson LH (2017) A PITX3-EGFP reporter line reveals connectivity of dopamine and non-dopamine neuronal subtypes in grafts generated from human embryonic stem cells. Stem Cell Reports 9, 868-882.

[24] Song B, Cha Y, Ko S, Jeon J, Lee N, Seo H, Park KJ, Lee IH, Lopes C, Feitosa M, Luna MJ, Jung JH, Kim J, Hwang D, Cohen BM, Teicher MH, Leblanc P, Carter BS, Kordower JH, Bolshakov VY, Kong SW, Schweitzer JS, Kim KS (2020) Human autologous iPSC-derived dopaminergic progenitors restore motor function in Parkinson's disease models. J Clin Invest 130, 904-920.

[25] Breton JM, Charbit AR, Snyder BJ, Fong PTK, Dias EV, Himmels P, Lock H, Margolis EB (2019) Relative contributions and mapping of ventral tegmental area dopamine and GABA neurons by projection target in the rat. J Comp Neurol 527, 916-941.

[26] Taylor SR, Badurek S, Dileone RJ, Nashmi R, Minichiello L, Picciotto MR (2014) GABAergic and glutamatergic efferents of the mouse ventral tegmental area. J Comp Neurol 522, 3308-3334.

[27] Root DH, Wang HL, Liu B, Barker DJ, Mod L, Szocsics P, Silva AC, Magloczky Z, Morales M (2016) Glutamate neurons are intermixed with midbrain dopamine neurons in nonhuman primates and humans. Sci Rep $\mathbf{6}$, 30615.

[28] Fisher LJ, Young SJ, Tepper JM, Groves PM, Gage FH (1991) Electrophysiological characteristics of cells within mesencephalon suspension grafts. Neuroscience $\mathbf{4 0}$, 109-122.

[29] Sorensen AT, Thompson L, Kirik D, Bjorklund A, Lindvall O, Kokaia M (2005) Functional properties and synaptic integration of genetically labelled dopaminergic neurons in intrastriatal grafts. Eur J Neurosci 21, 2793-2799.

[30] Adler AF, Bjorklund A, Parmar M (2020) Transsynaptic tracing and its emerging use to assess graft-reconstructed neural circuits. Stem Cells 38, 716-726.

[31] Grealish S, Heuer A, Cardoso T, Kirkeby A, Jonsson M, Johansson J, Bjorklund A, Jakobsson J, Parmar M (2015) 
Monosynaptic tracing using modified rabies virus reveals early and extensive circuit integration of human embryonic stem cell-derived neurons. Stem Cell Rep 4, 975-983.

[32] Antonini A, Poewe W, Chaudhuri KR, Jech R, Pickut B, Pirtosek Z, Szasz J, Valldeoriola F, Winkler C, Bergmann $\mathrm{L}$, Yegin A, Onuk K, Barch D, Odin P, co-investigators Gs (2017) Levodopa-carbidopa intestinal gel in advanced Parkinson's: Final results of the GLORIA registry. Parkinsonism Relat Disord 45, 13-20.

[33] Kamel WA, Al-Hashel JY (2020) LCIG in treatment of nonmotor symptoms in advanced Parkinson's disease: Review of literature. Brain Behav 10, e01757.

[34] Ghosh B, Zhang C, Ziemba KS, Fletcher AM, Yurek DM, Smith GM (2019) Partial reconstruction of the nigrostriatal circuit along a preformed molecular guidance pathway. $\mathrm{Mol}$ Ther Methods Clin Dev 14, 217-227.

[35] Wang Y, Tien LT, Lapchak PA, Hoffer BJ (1996) GDNF triggers fiber outgrowth of fetal ventral mesencephalic grafts from nigra to striatum in 6-OHDA-lesioned rats. Cell Tissue Res 286, 225-233.

[36] Lin L, Isacson O (2006) Axonal growth regulation of fetal and embryonic stem cell-derived dopaminergic neurons by Netrin-1 and Slits. Stem Cells 24, 2504-2513.

[37] Jasmin M, Ahn EH, Voutilainen MH, Fombonne J, Guix C, Viljakainen T, Kang SS, Yu LY, Saarma M, Mehlen P, Ye K (2021) Netrin-1 and its receptor DCC modulate survival and death of dopamine neurons and Parkinson's disease features. EMBO J 40, e105537.

[38] Gantner CW, de Luzy IR, Kauhausen JA, Moriarty N, Niclis JC, Bye CR, Penna V, Hunt CPJ, Ermine CM, Pouton CW, Kirik D, Thompson LH, Parish CL (2020) Viral delivery of GDNF promotes functional integration of human stem cell grafts in Parkinson's disease. Cell Stem Cell 26, 511-526 e515. 